\title{
Early Diagnosis and Management of Klebsiella Pneumonia Induced Panophthalmitis - A Case Report
}

\author{
Narayanan Balakrishnan ${ }^{1}$, Arvind Babu², Suhas Prabhakar ${ }^{3}$, Neha Arora ${ }^{4}$
}

\begin{abstract}
${ }^{1}$ Department of Ophthalmology, Sri Ramachandra Institute of Higher Education and Research, Chennai, Tamilnadu, India. ${ }^{2}$ Department of Ophthalmology, Sri Ramachandra Institute of Higher Education and Research, Chennai, Tamilnadu, India. ${ }^{3}$ Department of Ophthalmology, Sri Ramachandra Institute of Higher Education and Research, Chennai, Tamilnadu, India. ${ }^{4}$ Department of Ophthalmology, Sri Ramachandra Institute of Higher Education and
\end{abstract}

Research, Chennai, Tamilnadu, India

\section{INTRODUCTION}

Endophthalmitis is an inflammatory condition involving the intraocular cavities, including the Uveal tissue and retina. Panophthalmitis includes the sclera and Tenon's capsule. Endophthalmitis may be classified as Infective and Sterile endophthalmitis. Infective causes can be further subdivided into Exogenous, Endogenous and Secondary infections from surrounding structures. Exogenous forms arise from perforating injuries or postoperatively. Endogenous endophthalmitis may develop as a result of seeding from a distant focus of infection in the body. Depending on the causative organism, it may be bacterial or fungal.

Diabetes mellitus may accentuate the condition. Endophthalmitis can lead to serious complications such as visual loss and Phthisis bulbi. Hence if not managed adequately and early, enucleation may be required.

\section{PRESENTATION OF CASE}

A 55-year-old lady presented with complaints of fever and vomiting for 4 days with associated breathlessness. She also noticed diminution of vision in her left eye for 3 days, associated with discharge and pain. She had no prior history of any ocular trauma or surgeries. She was a known Diabetic for 15 years, on Oral medications and her sugars were uncontrolled. On general examination, she was found to be mildly tachycardic with a pulse rate of $102 \mathrm{bpm}$. There was diffuse abdominal tenderness, with no organomegaly. There were no other localising signs, other systems examination was normal.

On ocular examination, she had severe oedema of the left eyelid, with conjunctival chemosis. On slit lamp examination, the cornea was found to be mildly hazy due to endothelial pigments. A hypopyon was noted in the anterior chamber (AC), occupying $1 / 3^{\text {rd }}$ of the height of the AC with $4+$ cells. Pupillary reaction could not be determined due to the presence of hypopyon obscuring the view. She had a mature cataract and hence, posterior segment could not be visualised. IOP was $30 \mathrm{mmHg}$. There was restriction of extraocular movements in all directions. Examination of the right eye was found to be normal. [Table 1] [Image 1]

A B scan ultrasonography of the left eye demonstrated multiple vitreous echoes in the posterior vitreous cavity, with a diffuse choroidal thickening. Retina was noted to be on, and a T sign was seen on the posterior cavity, suggesting the possibility of panophthalmitis. [Image 2]
Corresponding Author:

Narayanan Balakrishnan, I/17 Plot No. 373, $11^{\text {th }}$ Street,

Anna Nagar, East Chennai-600102,

Tamilnadu, India.

E-mail: narayanbalakrishnan@hotmail.com

DOI: $10.14260 / \mathrm{jemds} / 2020 / 620$

How to Cite This Article:

Balakrishnan N, Babu A, Prabhakar S, et al. Early diagnosis and management of klebsiella pneumonia induced panophthalmitis- a case report. J Evolution Med Dent Sci 2020;9(38):2847-2849, DOI: $10.14260 /$ jemds/2020/620

Submission 24-03-2020,

Peer Review 14-05-2020,

Acceptance 20-05-2020,

Published 21-09-2020.

Copyright (C) $2020 \quad$ Narayanan Balakrishnan et al. This is an open access article distributed under Creative Commons Attribution License [Attribution 4.0 International (CC BY 4.0)] 
An MRI of the orbit was indicative of an infection involving the left ocular globe, the intraconal and extraconal space, the preseptal compartment and the anterior aspect of the soft tissues in the left temporal region. These findings were suggestive of the presence of endophthalmitis? scleritis, myositis, orbital and preseptal cellulitis with associated lacrimal adenitis. There was a small peripherally enhancing fluid collection in the preseptal compartment, which raised the suspicion of an abscess formation. Overall, the features were consistent with the clinical diagnosis of Panophthalmitis.

Systemic investigations were suggestive of a focal sepsis. [Table 2]. Ultrasonography showed a liver abscess, and a biopsy of the abscess also grew Klebsiella pneumoniae. An AC Tapping of the left eye was done, the sample was cultured and sent for XCYTON. It also grew Klebsiella pneumoniae. The pathogen was found to be susceptible to Amikacin, Amoxiclav, Cefoperazone with Sulbactam, Ciprofloxacin and Piperacillin with Tazobactam.

\section{DISCUSSION OF MANAGEMENT}

The patient was started on a course of systemic antibiotics with Intravenous Imipenem/Cilastatin and Piperacillin/ Tazobactam. Under strict aseptic precautions, three doses of Intravitreal injection Amikacin (0.4 mg / $0.1 \mathrm{~mL})$ and Ceftazidime (2.25 mg / $0.1 \mathrm{~mL}$ ) were given, starting on day 2 and followed up on day 4 and day 6 . Topical Tobramycin and Besifloxacin was applied 8 times a day in the left eye, along with Prednisolone eye drops $2^{\text {nd }}$ hourly and Cyclopentolate eye drops twice a day with Brimonidine/Timolol fixed combination to lower the IOP. A therapeutic AC tap was also done to relieve intraocular pressure. In view of severe chemosis and inflammation, she was started on oral NSAID's. The patient was counselled regarding the poor visual prognosis of the condition, and the possibility of evisceration was also contemplated.

After one week, her symptoms began to ameliorate and there was marked improvement in her systemic and ocular condition. Her left eye showed a significant reduction in discharge and inflammation with gradual resolution of the hypopyon. Intraocular pressure also returned to normal. [Image 3]

\begin{tabular}{|ccc|}
\hline Ocular Examination & Right Eye & Left Eye \\
Visual acuity & $6 / 6$ & Hand movement \\
Lids & WNL & Oedema \\
Conjunctiva & WNL & Chemosis \\
Cornea & Clear & Mildly hazy due to endothelial \\
pigments \\
Anterior chamber & Quite, normal depth & Cells 4+ hypopyon-1/3rd of AC \\
Pupil & Round, reacting to light & Not seen \\
Lens & Clear & Mature cataract \\
Fundus & WNL & No view \\
IOP (mmHg) & 16 & 30 \\
Extraocular movements & Full, free and painless & Restricted \\
\hline & Table 1 & \\
\hline & & \\
\hline
\end{tabular}

\begin{tabular}{|cc|}
\hline \multicolumn{2}{|c|}{ Systemic Investigations } \\
\hline Total leucocyte count & $24,300 / \mathrm{cu} . \mathrm{mm}$. \\
Polymorphs & $86 \%$ \\
Lymphocytes & $10 \%$ \\
Total bilirubin & $3.56 \mathrm{mg} / \mathrm{dL}$ \\
Direct bilirubin & $2.45 \mathrm{mg} / \mathrm{dL}$ \\
Alkaline phosphatase & $502 \mathrm{IU} / \mathrm{mL}$ \\
Blood sugars (RBS) & $380 \mathrm{mg} / \mathrm{dL}$ \\
\hline
\end{tabular}

\begin{tabular}{|cc|}
\hline Blood Culture & Klebsiella Pneumoniae \\
Liver biopsy & Klebsiella Pneumoniae \\
AC tap & Klebsiella Pneumoniae \\
\hline & Table 2 \\
\hline
\end{tabular}

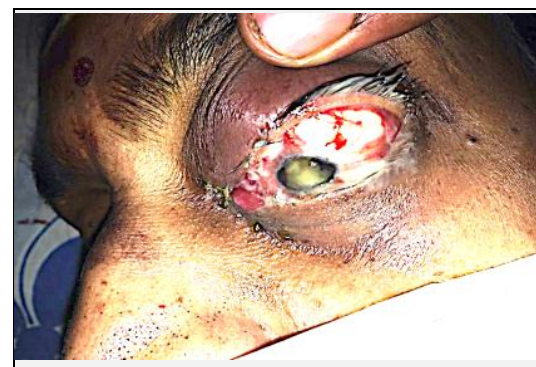

Figure 1.

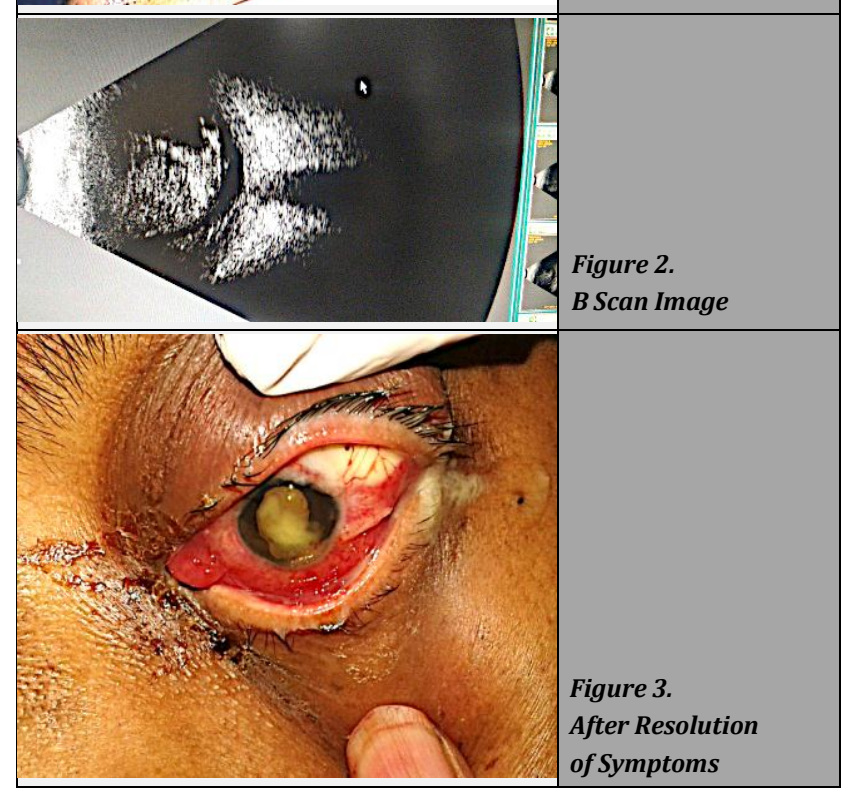

On 1 month follow up, there was complete resolution of inflammation and hypopyon. She then underwent a cataract surgery under the cover of topical steroids and antibiotics and visual acuity was restored.

A complete resolution of systemic symptoms was observed over the course of a week along with a significant reduction in ocular inflammation. Cataract surgery was carried out a month later under steroid cover leading to improvement of visual acuity as well.

\section{DISCUSSION}

Endogenous Klebsiella Endophthalmitis (EKE) is a devastating and potentially sight threatening ocular condition. It has been found that in the majority of cases, visual prognosis is grim despite aggressive use of antibiotics. Sridhar et al looked at Klebsiella endophthalmitis due to various causes, of which 3 had EKE. All patients eventually underwent an enucleation. They also reported the presence of Diabetes mellitus, Klebsiella induced pyogenic liver abscess and delayed treatment to be important risk factors for rapid progression and poor prognosis of EKE.[1] Sheu et al studied various risk factors for EKE and found uncontrolled diabetes to be associated with poor visual outcome in these patients. ${ }^{[2]}$ Yang et al also found Diabetes to be the most common risk factor and stated that early diagnosis and timely aggressive intervention are key to achieving a good visual outcome. 
Uncontrolled Diabetes was a risk factor present in our patient as well. Intravitreal injections if given promptly within 48 hours, have the potential to salvage the eye.[3] We initiated intravitreal injections within 36 hours of diagnosis and administered 3 doses in a timely manner, probably aiding in the resolution of symptoms. Treatment goals should also include reducing the intraocular pressure and external inflammation. The choice of intravitreal antibiotic is based on Vitreous/Anterior chamber culture, sensitivity and PCR. Fujita et al, treated 2 patients with EKE and associated pyogenic liver abscess. Both underwent eye surgery but eventually lost their vision due to delay in diagnosis and treatment. [4] In contrast, Karama et al reported a case of EKE in a diabetic patient with $K$. pneumoniae liver abscess, who was treated aggressively with systemic antibiotics and intravitreal antibiotic injections, which resulted in preservation of his visual acuity[5]. In our case, our patient had a similar presentation, with Klebsiella induced pyogenic liver abscess, with EKE, worsened by the presence of uncontrolled Diabetes. However, early diagnosis and aggressive systemic and ocular intervention resulted in a complete resolution of the condition. The importance of early Intravenous and intravitreal antibiotics in a setting such as this is paramount, without compromising on the systemic status of the patient. It is also important to stress that the diabetic status of the patient has to be monitored and the control of sugars is essential for systemic resolution.

Our case highlights that early management of both the systemic and ocular foci of infection can help in resolution of symptoms thus making it a rare case report contradicting previous claims that EKE complicated by a liver abscess leads to loss of the affected eye.

\section{CONCLUSIONS}

Diabetes is a significant risk factor for the development of endogenous endophthalmitis and poor visual outcome in $K$. pneumoniae liver abscess. Although the prognosis of endogenous K. pneumoniae endophthalmitis is generally poor, timely management as seen in this case can help in salvaging the patient's eyes. The prognosis of endogenous Klebsiella pneumoniae endophthalmitis is generally poor in a setting of diabetes with K. pneumoniae liver abscess.

Financial or Other Competing Interests: None.

\section{REFERENCES}

[1] Sridhar J, Flynn HW, Kuriyan AE, et al. Endophthalmitis caused by Klebsiella species. Retina 2014;34(9):1875-81.

[2] Sheu SJ, Kung YH, Wu TT, et al. Risk factors for endogenous endophthalmitis secondary to Klebsiella pneumoniae liver abscess: 20-year experience in Southern Taiwan. Retina 2011;31(10):2026-31.

[3] Yang CS, Tsai HY, Sung CS, et al. Endogenous Klebsiella endophthalmitis associated with pyogenic liver abscess. Ophthalmology 2007;114(5):876-80.

[4] Fujita M, Takahashi A, Imaizumi H, et al. Endogenous endophthalmitis associated with pyogenic liver abscess caused by Klebsiella pneumoniae. Intern Med 2019;58(17):2507-14.

[5] Karama EM, Willermain F, Janssens X, et al. Endogenous endophthalmitis complicating Klebsiella pneumoniae liver abscess in Europe: case report. Int Ophthalmol 2008;28(2):111-3. 